\title{
Medication administration error reporting and associated factors among nurses working at the University of Gondar referral hospital, Northwest Ethiopia, 2015
}

Berhanu Boru Bifftu ${ }^{1 *}$, Berihun Assefa Dachew², Bewket Tadesse Tiruneh and Debrework Tesgera Beshah ${ }^{1}$

\begin{abstract}
Background: Medication administration is the final step/phase of medication process in which its error directly affects the patient health. Due to the central role of nurses in medication administration, whether they are the source of an error, a contributor, or an observer they have the professional, legal and ethical responsibility to recognize and report. The aim of this study was to assess the prevalence of medication administration error reporting and associated factors among nurses working at The University of Gondar Referral Hospital, Northwest Ethiopia.

Methods: Institution based quantitative cross - sectional study was conducted among 282 Nurses. Data were collected using semi-structured, self-administered questionnaire of the Medication Administration Errors Reporting (MAERs). Binary logistic regression with $95 \%$ confidence interval was used to identify factors associated with medication administration errors reporting.

Results: The estimated medication administration error reporting was found to be $29.1 \%$. The perceived rates of medication administration errors reporting for non-intravenous related medications were ranged from 16.8 to 28. $6 \%$ and for intravenous-related from 20.6 to $33.4 \%$. Education status ( $\mathrm{AOR}=1.38,95 \% \mathrm{Cl}$ : 4.009, 11.128), disagreement over time - error definition ( $\mathrm{AOR}=0.44,95 \% \mathrm{Cl}: 0.468,0.990)$, administrative reason ( $\mathrm{AOR}=0.35,95 \% \mathrm{Cl}: 0.168,0.710$ ) and fear $(\mathrm{AOR}=0.39,95 \% \mathrm{Cl}: 0.257,0.838)$ were factors statistically significant for the refusal of reporting medication administration errors at $p$-value $<0.05$.

Conclusion: In this study, less than one third of the study participants reported medication administration errors. Educational status, disagreement over time - error definition, administrative reason and fear were factors statistically significant for the refusal of errors reporting at $p$-value $<0.05$. Therefore, the results of this study suggest strategies that enhance the cultures of error reporting such as providing a clear definition of reportable errors and strengthen the educational status of nurses by the health care organization.
\end{abstract}

Keywords: Errors reporting, Medication administration, Nurses

\footnotetext{
* Correspondence: berhanuboru@gmail.com

'Department of Nursing, University of Gondar College of Medicine and

Health Science, P. O. Box: 196, Gondar, Ethiopia

Full list of author information is available at the end of the article
} 


\section{Background}

Patient safety is the freedom from accidental or preventable injuries produced by medical care [1]. It is a central concern and an indicator of health care quality services [2]. That is why, concerns about patient safety have prompted the formation of a World Health Organization (WHO) patient safety program in response to a World Health Assembly in 2002 with the vision of 'every patient receives safe health care, every time, everywhere [3].

Patient safety can be affected as a result of a constellation of different factors and circumstances [2]. In all health care systems, medical errors are the main factors endangering the patient safety [4]. Medication errors are one of the most common types of medical errors. Medication errors are an error in the medication process: ordering, transcription, dispensing, and administration [5]. Medication errors listed in the top ten causes of mortality worldwide [6]. A systematic review of 45 studies revealed that the prevalence of medication errors ranges from 2 to $75 \%$ [7].

Medication administration error is one of the most common errors in the medication error process and occur when a discrepancy occurs between the drugs received by the patient and the drug intended by the prescriber [8]. For example, study from United Kingdom's National Patient Safety Agency revealed that of different types of medication errors, almost $50 \%$ account to administration error compared to $18 \%$ for dispensing, and $16 \%$ for prescribing [9]. A systematic literature review in Iran on medication error also indicated that reported prevalence of medication administration errors holds the highest ranges that is from 14.3 to $70 \%$ compared to $29.8-47.8 \%$ for prescribing error, $3-33.6 \%$ for dispensing error and from 10 to $51.8 \%$ for transcribing errors [10]. In Ethiopia, where there is lack of educated health care professionals and high patient flow, evidences indicate that medication error is a common problem that ranges from 4.35 to $89.9 \%[11,12]$. For example according to a recently published study, [13] the incidence of medication administration error was $56.4 \%$ of this, majority $(87.5 \%)$ indicated documentation error, followed by technique error $73.1 \%$ and time error 193 (53.6\%) respectively. Ages, work experience, nurse to patient ratio, medication administration at night shift were associated with errors [13].

In health care delivery system, the outcome of any errors result in serious patient outcomes [14] in terms of morbidity, mortality, adverse drug events, re-admissions to hospital, and increased length of hospital stay $[13,15,16]$. Therefore, prompt detection and report of an error can reduce the risk of such serious patient outcomes [17]. Voluntary medication error reporting systems rely on the ability and willingness of individual physicians, pharmacists, and nurses to detect and report errors as part of routine practice [18]. Nurses are intimately involved in and ultimately responsible for the delivery of medication [19]. The medication administration process is a daily component of nursing practice and is often viewed as a routine and basic nursing task that account for around $40 \%$ of their work time [15].

As part of the health care team, whether the nurses are the source of an error, a contributor, or an observer, they have a professional responsibility to recognize and report medication administration errors that could harm patient safety by clarifying ambiguous orders; and questioning orders that are inappropriate [14]. Error reporting through established systems provides opportunities to prevent future similar and perhaps even more serious errors, [9] nevertheless nurses are reluctant to report medication errors because of several reasons. Including: fear of disciplinary actions [20], lack of protection for reported errors, culture of blame and punishment, variations in how errors are defined and because of its potential damage to the hospitals' reputation $[4,7,9,18,20-22]$. Regarding the association of medication administration error reporting and nurses characteristics, most of the finding indicated there is no relationship between medication administration error reporting and nurses' characteristics. For example a survey of nurses revealed nurses did not vary in their concerns medication administration error reporting based on their socio demography such as age of the nurse, type of education and length of experiences [23, 24], married nurses and nurses on permanent contract [25]. Few study reveal inconsistent relation between medication administration error reporting and nurses' characteristics such as: experience, education, sex, working position, working unit $[26,27]$. Therefore, the main aim of this study was to assess medication administration error reported and associated factors among nurses working at the University of Gondar Hospital.

\section{Methods \\ Study design, periods and study area}

Institution based cross sectional quantitative study design was employed from May, 1 to 30, 2015 at The University of Gondar Hospital. The University of Gondar hospital is located at $748 \mathrm{~km}$ away from the capital city of Ethiopia, Addis Ababa. It is a tertiary level referral hospital, which acts as the referral centre for four district hospitals in the area and has 500 inpatient beds, and 559 health professionals to provide health service to the community of which the majority of them are nurses $(n=302)$. The hospital provides health referral services for over 5 million inhabitants in the North West region of Ethiopia.

\section{Participants}

The participants of this study were all nurses working in The University of Gondar Hospital. The inclusion criteria 
were those nurses who directly involved in patient care and had been employed for more than six months. Head nurses and those in higher administrative positions were excluded.

\section{Instruments}

For the assessment of medication administration errors reporting, we used medication administration errors reporting questionnaire. It contained 65 questions with three sections; the first section included 29 items regarding reasons why medication errors occur, second section included 16 items regarding reasons why medication errors not reported. Respondents were asked to indicate their level of agreement using a five point Likert type scale with fix values ranging from $5=$ strongly agree to $1=$ strongly disagree. The third section included 20 items regarding what percentage of each type of medication error actually reported in their units; 9 items for non intravenous (Non-IV) medication errors and 11 items for intravenous (IV) medication errors. More specifically, participants were asked to use a 10-point ordinal scale to indicate the range of MAEs which they perceived to be reported on their patient care units. This scale has been developed by Wakefield, et al., 2005. The items in the original instrument underwent rigorous validation and tested for its psychometric property factors analysis. The construct and criterion-related validity test and subscale reliability with Cronbach's alpha were ranging from .69 to $.76[24,28]$. This scale has been widely used across the world including Africa [22, 24]. In this study, the reliability of the questionnaire was measured by Cronbach's alpha and it had 0.81 for the 20 item MAER and 0.83 for the 16 item barrier to MAER.

For the purpose of this study, we used the estimated percentage of medication errors actually reported with 20 item and reasons why medication errors are not reported with16 items. The estimate of medication error reporting was defined by the estimated mean percentage of errors reported on the 20 items of medication errors that has actually been reported. This scale has been widely used across the world.

\section{Data collection methods}

Data were collected using a semi-structured self administered questionnaire consists of socio-demographic characteristics and medication administration errors reporting questionnaire. The questionnaires were distributed in the nursing office by two MSc nurses working in the academic area. The questionnaires need 10-20 min to complete.

\section{Data processing and analysis}

Data cleanup and cross-checking were made before the analysis. EPI info version 3.5.3 statistical software and Statistical Package for Social Science (SPSS) windows version 20 programs were used for data entry and analysis respectively. Bivariate and multivariate logistic regression and odds ratio with $95 \%$ confidence interval were used to identify the associated factors with medication administration errors reporting. Variables with $p$-value $(p<0.05)$ were used as the cutoff point.

\section{Results}

Total of 282 participants participated in this study with a $96.9 \%$ response rate.

\section{Socio-demographic characteristics of the respondents}

The majority of the participants were men 160 (56.7 \%). The mean ( \pm standard deviation) age of the participants was $28.89( \pm 9.70)$ years, $203(72 \%)$ of the participants were BSc in Nursing. Regarding their work experience, the majority of the participants 158 (56\%) served from 6 months to 4 years (Table 1 ).

\section{Perceived prevalence of medication administration error reporting}

The estimated medication administration error reported was found to be $29.1 \%$. The perceived rates of medication administration error reported for non intravenous medications were ranged from 18.1 to $28.4 \%$ and from 20.6 to $33.7 \%$ for intravenous related medication (Table 2).

\section{Reasons for Not reporting medication administration errors}

The overall mean and standard deviations of the subtotal scores were: $19 \pm 8.14$ for disagreement over time and error definition reasons, $12.03 \pm 5.37$ for fear reasons and $11.93 \pm 5.19$ for administrative reasons.

\section{Factors associated with medication administration error reporting}

From the bivariate analysis: sex, age, educational statuses, work experience, disagreement over time and error definition, administrative and fear reason were factors associated with the refusal of MAEs at $p$-value $<0.2$ and entered into multivariate analysis. From the multivariate analysis; Education status $(\mathrm{AOR}=1.38,95 \% \mathrm{CI}$ : $4.009,11.128)$, disagreement over time - error definition $(\mathrm{AOR}=0.44,95 \% \mathrm{CI}: 0.468,0.990)$, administrative reason $(\mathrm{AOR}=0.35,95 \% \mathrm{CI}: 0.168,0.710)$ and fear $(\mathrm{AOR}=0.39,95 \% \mathrm{CI}: 0.257,0.838)$ were factors statistically significant for the refusal of reporting medication administration errors at $p$-value $<0.05$ (Table 3).

\section{Discussion}

The main purpose of this study was to assess medication administration error reporting and associated factors. Finding from this study revealed that the estimated MAER was found to be $29.1 \%$. This finding support the 
Table 1 Socio-demographic characteristics of the respondents, Gondar University Referral Hospital (GURH), Northwest Ethiopia, $2015(n=282)$

\begin{tabular}{|c|c|c|}
\hline Characteristics & Number & Percent \\
\hline \multicolumn{3}{|l|}{ Sex } \\
\hline Male & 160 & 56.7 \\
\hline Female & 122 & 43.3 \\
\hline \multicolumn{3}{|l|}{ Age } \\
\hline $18-24$ & 44 & 15.6 \\
\hline $25-34$ & 190 & 67.4 \\
\hline $35-44$ & 33 & 11.7 \\
\hline$>=45$ & 15 & 5.3 \\
\hline \multicolumn{3}{|l|}{ Educational status } \\
\hline Diploma & 67 & 23.8 \\
\hline BSC in Nursing & 203 & 72 \\
\hline MSC in Nursing & 12 & 4.3 \\
\hline \multicolumn{3}{|l|}{ Religion } \\
\hline Orthodox & 242 & 85.8 \\
\hline Muslim & 30 & 10.6 \\
\hline Protestant & 7 & 2.5 \\
\hline Catholic & 3 & 1.1 \\
\hline \multicolumn{3}{|l|}{ Marital status } \\
\hline Single & 159 & 56.4 \\
\hline Married & 101 & 35.8 \\
\hline Divorced/Widowed & 22 & 7.8 \\
\hline \multicolumn{3}{|c|}{ Work experience in years } \\
\hline$\leq 4$ & 158 & 56 \\
\hline $5-10$ & 77 & 27.3 \\
\hline $11-14$ & 7 & 2.5 \\
\hline$>=15$ & 40 & 14.2 \\
\hline
\end{tabular}

study carried out in Korea and Taiwan that revealed the prevalence of $28.3 \%$ [29] and $24.5 \%$ [30] respectively. But the result of this study (29.1\%) is slightly higher than the study carried out in Saudi Arabia $22 \%$ [24]. On the other hand compared to other study carried out in Jordan, $86 \%$ [31] and $35 \%$ [32] and 48 to $70 \%$ in Taiwan [23], the result of this finding $(29.1 \%)$ is lower. The possible reason for the difference may be accounted for fear of legal issues, blame for the reported errors in the working environment and lack of personal confidence to withstand any punishments following the reporting of errors. Study from United State revealed that the main reasons for nurses do not report medication administration errors were: fear, disagreement over whether or not an error had occurred, administrative responses to medication errors, and the effort involved in the reporting process [33]. Another study also revealed that around $25 \%$ of the study participants did not reported error because of fear of their supervisors punish [34].

Regarding factor or barrier for MAER using MAER questionnaires, disagreement over time - error definition is considered as the most perceived barrier followed by fear reasons and administrative reasons for MAER. This finding is supported by several other studies using similar instrument as disagreement over time - error definition, fear reasons and administrative reasons are barrier for MAER with different ranking order [22, 23, 30, 31]. As to the statistical association, those participants who disagreed for the presence of disagreement over time error definition as a barrier for MAER were about forty four times more likely reported MAE (AOR $=0.44,95 \%$ CI:0.468, 0.990) than those participants who agreed for the presence of disagreement over time - error definition as a barrier for MAER. This finding is similar to other studies [35]. This could be due to the fact that those participants who do not clearly recognized the actual

Table 2 Proportion of perceived medication administration error reported for each item at GURH, Northwest Ethiopia, 2015 ( $n=282$ )

\begin{tabular}{|c|c|c|c|}
\hline \multicolumn{2}{|c|}{ Type of Medication Error } & \multicolumn{2}{|c|}{ Percentage of Each Type of Medication Error Actually Reported } \\
\hline & & \multirow{2}{*}{$\begin{array}{l}\text { Intra Venous (IV) Error } \\
20.6\end{array}$} & \multirow{2}{*}{$\frac{\text { Non-Intra Venous (IV) Erro }}{26.2}$} \\
\hline 1 & Wrong route & & \\
\hline 2 & Wrong time & 33.3 & 27.7 \\
\hline 3 & Wrong patient & 20.6 & 18.8 \\
\hline 4 & Wrong Dose & 30.5 & 19.1 \\
\hline 5 & Wrong drug & 28.4 & 18.8 \\
\hline 6 & Medication is omitted. & 30.1 & 28.4 \\
\hline 7 & Medication is given, but not ordered by physician. & 20.9 & 24.8 \\
\hline 8 & Medication administered after the order discontinued. & 33.7 & 23.4 \\
\hline 9 & Given to patients with a known allergy. & 22.7 & 18.1 \\
\hline 10 & Wrong fluid & 22.3 & - - \\
\hline 11 & Wrong rate & 23.8 & -— \\
\hline
\end{tabular}


Table 3 Bivariate and multivariate logistic regression analysis of factors associated with MAER at GURH, Northwest Ethiopia, 2015 ( $n=282)$

\begin{tabular}{|c|c|c|c|c|c|}
\hline \multirow[t]{2}{*}{ Explanatory variables } & \multicolumn{2}{|l|}{ MAER } & \multirow[t]{2}{*}{ COR $(95 \% \mathrm{Cl})$} & \multirow[t]{2}{*}{ AOR (95 \% Cl) } & \multirow[t]{2}{*}{$P$-value } \\
\hline & Yes N (\%) & No N (\%) & & & \\
\hline \multicolumn{6}{|l|}{ Sex } \\
\hline Male & $38(23.8)$ & $122(76.2)$ & $0.55(1.078,3.043)$ & & \\
\hline Female & $44(36.1)$ & $78(63.9)$ & 1 & & \\
\hline \multicolumn{6}{|l|}{ Age } \\
\hline $20-24$ & $10(22.7)$ & $34(77.3)$ & 1 & & \\
\hline $25-34$ & $52(27.4)$ & $138(72.6)$ & $1.28(0.360,1.1 .692)$ & & \\
\hline $35-44$ & $15(45.5)$ & $18(54.5)$ & $2.83(1.132,5.943)$ & & \\
\hline$>/=45$ & $5(33.3)$ & $10(66.7)$ & $1.7(0.163,2.125)$ & & \\
\hline \multicolumn{6}{|l|}{ Educational status } \\
\hline Diploma & $11(16.4)$ & $56(83.6)$ & 1 & 1 & \\
\hline BSc and above & $71(33)$ & $144(67)$ & $2.51(2.188,9.775)$ & $1.38(4.009,11.128)$ & $<0.001$ \\
\hline \multicolumn{6}{|c|}{ Work experience in year } \\
\hline$</=4$ & $39(24.7)$ & $119(75.3)$ & 1 & & \\
\hline $5-10$ & $23(29.9)$ & $54(70.1)$ & $1.30(0.419,1.412)$ & & \\
\hline $11-15$ & $5(71.4)$ & $2(28.6)$ & $7.63(0.024,0.703)$ & & \\
\hline$>/=16$ & $15(37.5)$ & $25(62.5)$ & $1.83(0.262,1.139)$ & & \\
\hline \multicolumn{6}{|c|}{ Disagreement over time - error definition } \\
\hline Agree & $38(33.1)$ & $89(66.9)$ & 1 & 1 & 1 \\
\hline Disagree & $44(25)$ & $111(75)$ & $0.93(0.201,0.778)$ & $0.44(0.468,0.990)$ & $<0.001$ \\
\hline \multicolumn{6}{|l|}{ Administrative reason } \\
\hline Agree & $47(33.1)$ & $95(66.9)$ & 1 & 1 & 1 \\
\hline Disagree & $35(25)$ & $105(75)$ & $0.67(0.401,0.978)$ & $0.35(0.168,0.710)$ & 0.004 \\
\hline \multicolumn{6}{|l|}{ Fear reason } \\
\hline Agree & $40(26)$ & $87(74)$ & 1 & 1 & 1 \\
\hline Disagree & 43 (31.6) & $112(68.4)$ & $0.84(0.451,0.767)$ & $0.39(0.257,0.838)$ & 0.006 \\
\hline
\end{tabular}

definition of medication administration error do not report the error because reportable errors need their own knowledge like the knowledge of error, who it could be reported, to whom it reported and the like. Studies support this explanation as a knowledge deficit is seen in lack of knowledge of process (not knowing how to report an error) and uncertainty about definitions (e.g., what is an error? What is a near miss?) for the refusal to report errors [22, 36]. Another cross-sectional study from Korea revealed that although $95 \%$ of participants were not afraid to report mistakes, but about half of the respondents reported that they were not clear about the types of errors should be reported [37].

Those participants who disagreed administrative reason as a barrier for MAER were thirty five times more likely reported MAE $(\mathrm{AOR}=0.35,95 \% \mathrm{CI}: 0.168,0.710)$ than those participants who agreed administrative reason as barrier for MAER. In this study more than half (50\%) of the study participant did not reported MAE because of Administrative Reason. This is similar with other studies [22, 23, 37].

Those participants who disagreed fear reason as a barrier for MAER were about thirty nine times $(\mathrm{AOR}=0.39$, 95 \% CI: $0.257,0.838$ ) more likely reported MAE than those participants who agreed fear reason as barrier for MAER. This may be due to the fact that fear of the consequence of the errors on their future career. This result is consistent with other studies [16, 22, 23, 30,31].

Those participants who had educational status of BSc and above were more than one times (AOR $=1.38,95 \%$ CI: $4.009,11.128)$ more likely reported MAE than those participants who had educational status of diploma. These result is consistent with the previous study. This is due to the fact that those participants who had higher educational status may have higher knowledge, attitude and practice toward the drug adverse effect or they may develop confidence to defend the consequence of MAER through their educational journey. 


\section{Limitation of the study}

This study has some important limitations that should be kept in mind when interpreting the results. The cross-sectional nature of the study design does not confirm definitive cause and effect relationship and since, the study was based on self-reported information that may be prone for reporting bias because of the respondent's interpretation of the questionnaire or desire to report their feeling. Some nurses characteristics such as working area (unit), working position and other were not collected that may affect MAE reporting.

\section{Conclusion}

Overall, more than two third of the participants did not reported medication administration errors. Educational status, disagreement over time - error definition, administrative and fear reason were factors statistically significant for the refusal of reporting medication administration errors at $p$-value $<0.05$. Therefore, the results of this study suggest strategies that enhance the cultures of error reporting such as providing a clear definition of reportable errors, establishing a good relationship with the healthcare administrators that make the workers free to report any mistakes without fear and strengthen the educational status of nurses by the health care organization.

\section{Abbreviations}

AOR, adjusted odd ratio; $\mathrm{Cl}$, confidence interval; COR, crude odd ratio; MAER, Medication Administration Error Reporting; Non-IV, non intravenous; SPSS, statistical package for social science; WHO, World Health Organization

\section{Acknowledgment}

The authors would like to thank The University of Gondar for the financial support to conduct this research. Funder had no role in study design, data collection, analysis and decision to publish. The corresponding author had full access to all the data in this study and had final responsibility for the decision to prepare the manuscript and submit for publication. We would also like to thank the study subjects for their willingness to participate in the study to share their experience.

\section{Funding}

The fund of this paper was covered by University of Gondar College of Medicine and Health Science. Funder had no role in study design, data collection, analysis and decision to publish. The corresponding author had full access to all the data in the study and had final responsibility for the decision to prepare the manuscript and submit for publication.

\section{Availability of data and materials}

Data can be provided on request from the corresponding author on reasonable request.

\section{Authors' contribution}

BB carried out the manuscript from its outset, analysis and interpretation of data and drafted the manuscript. BA, BT and DT participated in data analysis, interpretation and review of the manuscript. All authors read and approved the final version of manuscript.

\section{Authors' information}

BB, BT and DT are from The University of Gondar College of Medicine and Health Science, Department of Nursing, Gondar, Ethiopia. BA from The University of Gondar College of Medicine and Health Science, Institute of Public Health, Department of Epidemiology and Biostatistics, Gondar, Ethiopia.

\section{Competing interests}

The authors declare that they have no competing interests.

Consent for publication

Not applicable.

\section{Ethics approval and consent to participate}

The study proposal was approved by the ethical review board of The University of Gondar and a formal letter of permission was obtained. Verbal and then written informed consent was sought from each participant who agreed to participate in the study.

\section{Author details}

${ }^{1}$ Department of Nursing, University of Gondar College of Medicine and Health Science, P. O. Box: 196, Gondar, Ethiopia. ${ }^{2}$ Department of Epidemiology and Biostatistics, Institute of Public Health, University of Gondar College of Medicine and Health Science, P. O. Box: 196, Gondar, Ethiopia.

Received: 18 December 2015 Accepted: 13 July 2016

Published online: 18 July 2016

\section{References}

1. Quality AfHRa. Preparing and Analyzing Data and Producing Report. 2008. Retrieved on February.

2. Westat R, Joann, S. and Veronica, N. Hospital survey on patient safety culture; Agency for healthcare research and quality US Department of Health and Hu-man Services AHRQ. http://www.ahrq.gov.004.

3. Organization WH. Patient Safety Program in Response to a World Health Assembly. 2002

4. Stratton KM, Blegen MA, Pepper G, Vaughn T. Reporting of medication errors by pediatric nurses. J Pediatr Nurs. 2004;19(6):385-92.

5. Fontan J, Maneglier V, Nguyen VX, Loirat C, Brion F. Medication errors in hospitals: computerized unit drug dispensing systems versus ward stock distribution system. Pharm World Sci. 2003;25(3):112-7.

6. Mahajan RP. Medication errors: can we prevent them? BJA. 2011;107(1):3-5.

7. Lisby M, Nielsen LP, Brock B, et al. How are medication errors defined? A systematic literature review of definitions and characteristics. Int J Qual Health Care. 2010;22:507-18.

8. Flynn L, Liang Y, Dickson GL, et al. Nurses' practice environments, error interception practices, and inpatient medication errors. J Nurs Scholarsh. 2012:44:180-6.

9. Schmidt CE, Bottoni T. Improving medication safety and patient care in the emergency department. J Emerg Nurs. 2003;29:12-6.

10. Ahmadizar F, Soleymani F, Abdollahi M. Study of drug-drug interactions in prescriptions of general practitioners and specialists in Iran 2007-2009. Iran J Pharm Res. 2011;10:921-31.

11. Negash G, Kebede Y, Hawaze S. Medication errors in the adult emergency unit of a tertiary care teaching hospital in Addis Ababa. Arch Pharm Pract. 2013;4(4):147-50.

12. Girma B, Feleke Y. Medication Administration Errors Involving Paediatric In-Patients in a Hospital in Ethiopia. Trop J Pharm Res. 2010;9(4):401-7.

13. Feleke SA, Mulato MA, Yesmaw YS. Medication administration error: magnitude and associated factors among nurses in Ethiopia. BMC Nurs. 2015;14(53):1-8

14. Runciman WB, Sellen A, Webb RK, et al. Errors, incidents and accidents in anesthetic practice. Anesth Intensive Care. 1993;21:506-19.

15. Armitage $\mathrm{G}$, Knapman $\mathrm{H}$. Adverse events in drug administration: a literature review. J Nurs Manag. 2003;11:130-40.

16. Aboshaiqah AE. Nurses' perception of medication administration errors. Am J Nurs Res. 2014;2(4):63-7.

17. Bates DW, Leape LL, Petrycki S. Incidence and preventability of adverse drug events in hospitalized adults. J Gen Intern Med. 1993;8:289-94.

18. Flynn EA, Pepper GA, et al. Comparison of methods for detecting medication errors in 36 hospitals and skilled-nursing facilities. Am J Health Syst Pharm. 2002;59(5):436-46.

19. Carlton G, Blegen MA. Medication-related errors: a literature review of incidence and antecedents. Annu Rev Nurs Res. 2006;24:19-38.

20. Bahadori M, Ravangard R, Aghili A, Sadeghifar J, Manshadi MG, Smaeilnejad $J$. The factors affecting the refusal of reporting on medication errors from 
the Nurses' viewpoints:a case study in a hospital in Iran. Hindawi Publishing Corporation. ISRN Nursing. 2013;2013:5.

21. Gladstone J. Drug administration errors: a study into the factors underlying the occurrence and reporting of drug errors in a district general hospital. J Adv Nurs. 1995;22:628-37.

22. MI-AE YOU1 M-H. Perceptions regarding medication administration errors among hospital staff nurses of South Korea. International Journal for Quality in Health Care. 2015;27(4):276-83.

23. Chiang HY, Lin SY, Hsu SC, Ma SC. Factors determining hospital nurses' failures in reporting medication errors in Taiwan. Nurs Outlook. 2010;58:17-25.

24. Suliman Ali Al-Youssif LKM, Mohamed NS. Nurses' experiences toward perception of medication administration errors reporting. IOSR J Nurs Health Scienc. 2013;1(4):56-70.

25. Sara Rizvi Jafree RZ, Zakar MZ, Fischer F. Nurse perceptions of organizational culture and its association with the culture of error reporting: a case of public sector hospitals in Pakistan. BMC Health Serv Res. 2016;16(3):1-13.

26. Easton K, Morgan T, Williamson M. Medication Safety in the Community: A Review of the Literature. Sydney: National Prescribing Service; 2009.

27. Fawzia El Sayed Abusaad EAE. Medication administration errors at Children's University hospitals: nurses point of view. J Nur Health Sci. 2015;4(1):51-60.

28. Wakefield BJ, Uden-Holman T, Wakefiled DS. Development and validation of the medication administration error reporting survey. Adv Patient Saf. 2005; 4:475-89.

29. Kim KS, Kwon SH, Kim JA, et al. Nurses' perceptions of medication errors and their contributing factors in South Korea. J Nurs Manag. 2011;19:346-53.

30. Chiang H-YaP GA. Barriers to Nurses' reporting of medication administration errors in Taiwan. J Nurs Scholarsh. 2006;38:392-9.

31. IG Al-Faouri, WA. Hayajneh, DM Habboush. A Five Years Retrospective Study of Reported Medication Incidents at a Jordanian Teaching Hospital: Patterns and Trends. International Journal of Humanities and Social Science. 2014; 4(5)280-287.

32. Mrayyan MT. Reported incidence, causes, and reporting of medication errors in teaching hospitals in Jordan: a comparative study. Contemp Nurse. 2012; 41(2):216-32.

33. Wakefield DS, Wakefield BJ, Uden-Holman T, et al. Perceived barriers in reporting medication administration errors. Best Practice in Benchmarking Healthcare. 1996;1:191-7.

34. Elnitsky C, Nichols B, Palmer K. Are Hospital Incidents being reported? J Nurs Adm. 1997;27:40-6.

35. El-Bialy GG, Hashish EAA. Nurses' perceptions of safety climate and barriers to report medication errors. Life Sci J. 2013;10(1):2160-8.

36. Mostafaei D, Barati Marnani A, Mosavi Esfahani H, Estebsari F, Shahzaidi S, Jamshidi E, Aghamiri SS. Medication errors of nurses and factors in refusal to report medication errors among nurses in a teaching medical center of Iran. Iran Red Crescent Med J. 2014;16(10):1-6.

37. Kim J, An K, Kim MK, Yoon SH. Nurses' perception of error reporting and patient safety culture in Korea. West J Nurs Res. 2007;29:827.

\section{Submit your next manuscript to BioMed Central and we will help you at every step:}

- We accept pre-submission inquiries

- Our selector tool helps you to find the most relevant journal

- We provide round the clock customer support

- Convenient online submission

- Thorough peer review

- Inclusion in PubMed and all major indexing services

- Maximum visibility for your research

Submit your manuscript at www biomedcentral.com/submit

) Biomed Central 